\title{
Effects of severe mental illness on survival of people with diabetes
}

\author{
Yana Vinogradova, Carol Coupland, Julia Hippisley-Cox, Seán Whyte and Catherine Penny
}

\section{Background}

People with mental health problems are more likely to die prematurely than the general population but no study has examined this in individuals with diabetes.

\section{Aims}

To compare survival rates in people with diabetes with and without schizophrenia or bipolar disorder.

\section{Method}

A total of 43992 people with diabetes were drawn from the QRESEARCH database population of over 9 million patients. Survival rates during the study period, between 1 April 2000 and 1 April 2005, and hazard ratios for deaths associated with schizophrenia and bipolar disorder were adjusted by age and gender and additionally for socioeconomic status, obesity, smoking and use of statins.

\section{Results}

Among the participants, we identified 257 people diagnosed with schizophrenia, 159 with bipolar disorder and 14 with

both conditions. Although crude survival rates did not show significant differences between the groups during the study period, people with schizophrenia or bipolar disorder and diabetes, compared with those with diabetes alone, had a significantly increased risk of death after adjusting for age and gender, with hazard ratios for schizophrenia of 1.84 (95\% $\mathrm{Cl} 1.42-2.40)$ and for bipolar disorder of 1.51 (95\% Cl 1.102.07). After adjusting for the other factors, hazard ratios were 1.52 (95 Cl 1.17-1.97) for schizophrenia and $1.47(95 \% \mathrm{Cl}$ 1.07-2.02) for bipolar disorder.

\section{Conclusions}

People with schizophrenia or bipolar disorder in addition to diabetes have a relatively higher mortality rate. This suggests that diabetes either progresses more rapidly or is more poorly controlled in these individuals, or that they have higher levels of comorbidity and so are more likely to die of other causes.

\section{Declaration of interest}

None.
People with mental disorders are considerably more likely to die prematurely than the general population. In a systematic review, Harris \& Barraclough ${ }^{1}$ found that the mortality rate remained higher in individuals with mental disorders when deaths from unnatural causes (suicide and violent death) were excluded. In people with schizophrenia, the standardised mortality ratio (SMR) for all natural causes was $137 \%$ (95\% CI 134-141\%) compared with $100 \%$ for a general population of similar age and gender, accounting for $62 \%$ of the excess deaths from all causes. Most excess deaths were from infectious, respiratory and digestive system disorders, but deaths from endocrine, circulatory and genitourinary system disorders also had significantly raised SMRs. The SMR for endocrine system disorders, including diabetes, was $238 \%$ (95\% CI 114-438\%). In people with bipolar disorder, the SMR for all natural causes was 150\% (95\% CI $137-164 \%$ ), although only deaths from circulatory and respiratory system disorders had significantly raised SMRs.

A subsequent study ${ }^{2}$ found an SMR of 260 (95\% CI 219-306) for all natural causes in people with schizophrenia, mainly as a result of diseases of the circulatory, digestive, endocrine, nervous and respiratory systems, with an SMR of 801 (95\% CI 322-1651) for endocrine system disorders. Studies from two states in the $\mathrm{USA}^{3,4}$ found the life expectancy of people with severe mental illness to be about 9 years lower than that of the general population.

The association between severe mental illness and diabetes is now widely recognised. A consensus meeting in 2003 concluded that the overall risk of type 2 diabetes in people with schizophrenia is between two and four times that of the general population, with a prevalence of approximately $15-18 \%$, and that impaired glucose tolerance may affect up to $30 \%$ of people with schizophrenia. ${ }^{5}$ Similar findings have been reported in people with bipolar disorder. ${ }^{6}$

A study in the USA examined the impact of diabetes on mortality in 197 individuals with co-occurring psychotic and substance use disorders participating in a randomised controlled study of integrated mental health and substance misuse treatment. The study found that participants with evidence of diabetes were significantly more likely to die during follow-up than participants without evidence of diabetes. ${ }^{7}$ However, no study has yet demonstrated this in a naturalistic population sample. This study examines mortality rates in a cohort of people with diabetes, comparing those with and without schizophrenia and bipolar disorder. It tests the hypothesis that having one of these mental illnesses increases the risk of premature death in individuals with diabetes in a large and representative primary care population sample.

\section{Method}

\section{Participants}

The study used the QRESEARCH database version 8 (www. qresearch.org/), which is derived from the computerised health records of general practices using the Egton Medical Information System (EMIS) medical record computer system (Egton Medical Information Systems Limited, Leeds, UK). The full database at the time of the study contained aggregated data on more than 9 million patients from 525 representative general practices across the UK and includes information on patient demographics, diagnoses, clinical values, laboratory investigations, prescriptions, consultations and referrals. The database has been 
validated by comparing birth and death rates, consultation rates and prevalence and mortality rates with other data sources, ${ }^{8}$ and has demonstrated good levels of completeness and consistency. ${ }^{9}$

\section{Inclusion criteria}

QRESEARCH practices were eligible for inclusion in the study if their current EMIS computer system was installed before 1 April 1999 and they had complete data from then until 1 April 2005. Individuals from these practices were included if they: had a diagnosis of diabetes recorded between 1 January 1990 and 1 April 2000; were alive and registered with an eligible practice on 1 April 2000 (the study entry date for these analyses); had been registered with an eligible practice for at least the previous 12 months; were 25 years or older on 1 April 2000; and were not registered as a temporary resident.

\section{Diagnoses}

Individuals' diagnoses for diabetes, schizophrenia and bipolar disorder were determined using the standard computer codes (Read codes) for general practice in the UK. These codes are entered by general practitioners (GPs) when they make their clinical records of a consultation. A full list of codes, developed in consultation with GPs with an interest in the field, is available from the authors. Diagnoses were included whether they were coded as active or inactive, current or past, because the 'inactive' and 'past' codes were not reliably or consistently used by those entering data.

Type 1 and type 2 diabetes were recorded and analysed together, because the recording of diabetes in primary care does not always distinguish between types. Smoking status and obesity were defined according to the last recorded status of the individual prior to 1 April 2000. Diagnoses of schizophrenia and bipolar disorder were defined if they were recorded before 1 April 2000. Data on deaths from all causes between 1 April 2000 and 1 April 2005 were extracted from the records.

\section{Primary outcomes and analysis}

The overall survival rates for people with diabetes were determined, comparing those with and without schizophrenia and those with and without bipolar disorder. Where people had diagnoses of schizophrenia and bipolar disorder, they contributed to the analyses of both groups. The date of diagnosis of diabetes was used as the time origin in all analyses, with 1 April 2000 defined as the delayed entry date. The proportions of people alive 5 and 10 years after being diagnosed with diabetes were also determined for each population subgroup using Kaplan-Meier estimators and the differences between the groups were assessed with the log-rank test.

To estimate the risk of death in individuals with each mental disorder compared with those without, a Cox regression survival analysis was performed adjusted for age at diagnosis of diabetes (in years) and gender, and further for smoking status (smoker, non-smoker, not recorded) and body mass index $\left(<25 \mathrm{~kg} / \mathrm{m}^{2}\right.$, $25-29 \mathrm{~kg} / \mathrm{m}^{2}, \geqslant 30 \mathrm{~kg} / \mathrm{m}^{2}$; not recorded). As it has been shown that people with schizophrenia and bipolar disorders are prescribed statins less often than the rest of the population, ${ }^{10}$ use of statins was included. The analysis was also adjusted for Townsend deprivation score (in fifths, with higher values indicating greater deprivation), which is based on 2001 postcode-related census data, reflecting unemployment, overcrowding, lack of home ownership and lack of car ownership, and is associated with mortality in the general population. ${ }^{11}$ In an additional analysis we also adjusted for antipsychotic use. The baseline characteristics of people with each mental disorder were compared with those without mental illness using Student's $t$-test or the chi-squared test depending on the distribution of the characteristic. All analyses were conducted in STATA version 10 for Windows.

\section{Results}

A total of 43992 people with diabetes from 372 practices met all the inclusion criteria for the analysis. At those practices, there were a total of 1896944 patients aged 25 or over on the study entry date who had been permanently registered for at least 12 months, giving a prevalence of diabetes in those aged 25 and over of $2.3 \%$. Of the 43992 people with diabetes, $257(0.58 \%)$ had a diagnosis of schizophrenia, $159(0.36 \%)$ had a diagnosis of bipolar disorder and 14 were diagnosed with both conditions (these individuals are also included in the 257 and 159 figures above). These proportions are broadly comparable with other UK population surveys. ${ }^{12,13}$

Table 1 and Fig. 1 show the characteristics of the individuals with schizophrenia, bipolar disorder and with neither condition. The proportion of women was higher in both mental illness groups: $55 \%$ of individuals with schizophrenia were female and $60 \%$ of those with bipolar disorder were female compared with $46 \%$ of those with diabetes alone. Participants in both mental illness groups were less likely to live in rural areas, more likely to live in deprived areas (Townsend score fifths 4 or 5) and to smoke, and - for individuals with schizophrenia - to have a higher body mass index. Individuals in both mental illness groups had a lower mean systolic blood pressure.

People with schizophrenia were younger at study entry and when diagnosed with diabetes. In total, $48 \%$ were aged 60 years or older at diagnosis compared with $59 \%$ of those without schizophrenia or bipolar disorder. Use of statins in the 12 months prior to the study reference date was lower in individuals with both schizophrenia and diabetes than in the population with diabetes alone ( $10 \%$ v. $17 \%)$.

\section{Survival rates}

During the study period, 8698 people died. Of these, 57 had schizophrenia, 39 had bipolar disorder and 1 had both conditions. In total, $22 \%$ of those with schizophrenia and $25 \%$ of those with bipolar disorder died during follow-up compared with $20 \%$ with diabetes alone.

Table 2 shows 5- and 10-year survival rates for individuals with diabetes and schizophrenia or bipolar disorder and with diabetes alone; these are estimates of the proportions of individuals who are still alive 5 years and 10 years after being diagnosed with diabetes. Although people with these mental health problems had lower survival rates than individuals with diabetes alone, the differences in the survival rates were not statistically significant for either schizophrenia or bipolar disorder.

\section{Hazard ratios}

Table 3 presents the hazard ratios for dying in those with diabetes, comparing individuals with and without schizophrenia and bipolar disorder. The adjusted hazard ratios show the risks taking into account the differences in age and gender and also the differences in deprivation, obesity, smoking habits and use of statins between the groups. Whereas the unadjusted hazard ratios do not reach statistical significance, the adjusted hazard ratios are significantly increased for people with schizophrenia and those with bipolar disorder compared with those with diabetes alone. 
Table 1 Characteristics of individuals with diabetes: numbers (\%) and means (s.d.) in the groups with schizophrenia and bipolar disorder and without these mental illnesses

\begin{tabular}{|c|c|c|c|}
\hline & $\begin{array}{l}\text { With schizophrenia } \\
\qquad(n=257)\end{array}$ & $\begin{array}{l}\text { With bipolar disorder } \\
\qquad(n=159)\end{array}$ & $\begin{array}{l}\text { No schizophrenia or bipolar disorder } \\
\qquad(n=43589)\end{array}$ \\
\hline Female, $n$ (\%) & $141(54.9)^{*}$ & $96(60.4)^{*}$ & 19854 (45.5) \\
\hline Male, $n(\%)$ & $116(45.1)^{*}$ & $63(39.6)^{*}$ & $23736(54.5)$ \\
\hline Age on 1 April 2000, years: mean (s.d.) & $60.9(12.5)^{*}$ & $64.5(13.0)$ & $65.2(13.8)$ \\
\hline Body mass index, $\mathrm{kg} / \mathrm{m}^{2}$ : mean (s.d.) & $29.8(6.4)^{*}$ & $30.0(6.2)$ & $29.0(5.8)$ \\
\hline Systolic blood pressure, mmHg: mean (s.d.) & $138.4(21.0)^{\star}$ & $139.2(19.3)^{*}$ & $144.8(20.1)$ \\
\hline Diastolic blood pressure, mmHg: mean (s.d.) & $80.9(11.3)$ & $80.5(9.1)$ & $81.2(10.1)$ \\
\hline Age at diagnosis of diabetes & * & & \\
\hline Age, years: mean (s.d.) & $57.2(12.6)^{*}$ & $60.4(13.1)$ & $61.1(13.9)$ \\
\hline Under 50 years, $n(\%)$ & $67(26.1)$ & $30(18.9)$ & $8563(19.6)$ \\
\hline 50-59 years, $n(\%)$ & $68(26.5)$ & $34(21.4)$ & 9566 (21.9) \\
\hline $60-69$ years, $n(\%)$ & $82(31.9)$ & $58(36.5)$ & $12666(29.1)$ \\
\hline 70 years plus, $n(\%)$ & $40(15.6)$ & $37(23.3)$ & $12795(29.4)$ \\
\hline Townsend score: fifths, $n$ (\%) & * & * & \\
\hline 1 (least deprived) & $19(7.4)$ & $20(12.6)$ & $7927(18.2)$ \\
\hline 2 & $26(10.1)$ & $21(13.2)$ & $7853(18.0)$ \\
\hline 3 & $34(13.2)$ & $22(13.8)$ & $8342(19.1)$ \\
\hline 4 & 49 (19.1) & $40(25.2)$ & 8477 (19.4) \\
\hline 5 (most deprived) & $120(46.7)$ & $45(28.3)$ & $8969(20.6)$ \\
\hline Lives in rural area & $52(20.2)^{\star}$ & $37(23.3)^{\star}$ & $15165(34.8)$ \\
\hline Smoking status, $n$ (\%) & * & * & \\
\hline Smoker & $84(32.7)$ & $46(28.9)$ & $7054(16.2)$ \\
\hline Non-smoker & $132(51.4)$ & $103(64.8)$ & $32739(75.1)$ \\
\hline Not recorded & $41(16.0)$ & $10(6.3)$ & $3796(8.7)$ \\
\hline \multicolumn{4}{|l|}{ Medication, $n(\%)$} \\
\hline On antipsychotics prior to study period & $168(65.4)^{*}$ & $68(42.8)^{*}$ & 4925 (11.3) \\
\hline On statins prior to study period & $26(10.1)^{*}$ & $24(15.1)$ & 7561 (17.3) \\
\hline Died during study period, $n$ (\%) & $57(22.2)$ & $39(24.5)$ & $8603(19.7)$ \\
\hline
\end{tabular}

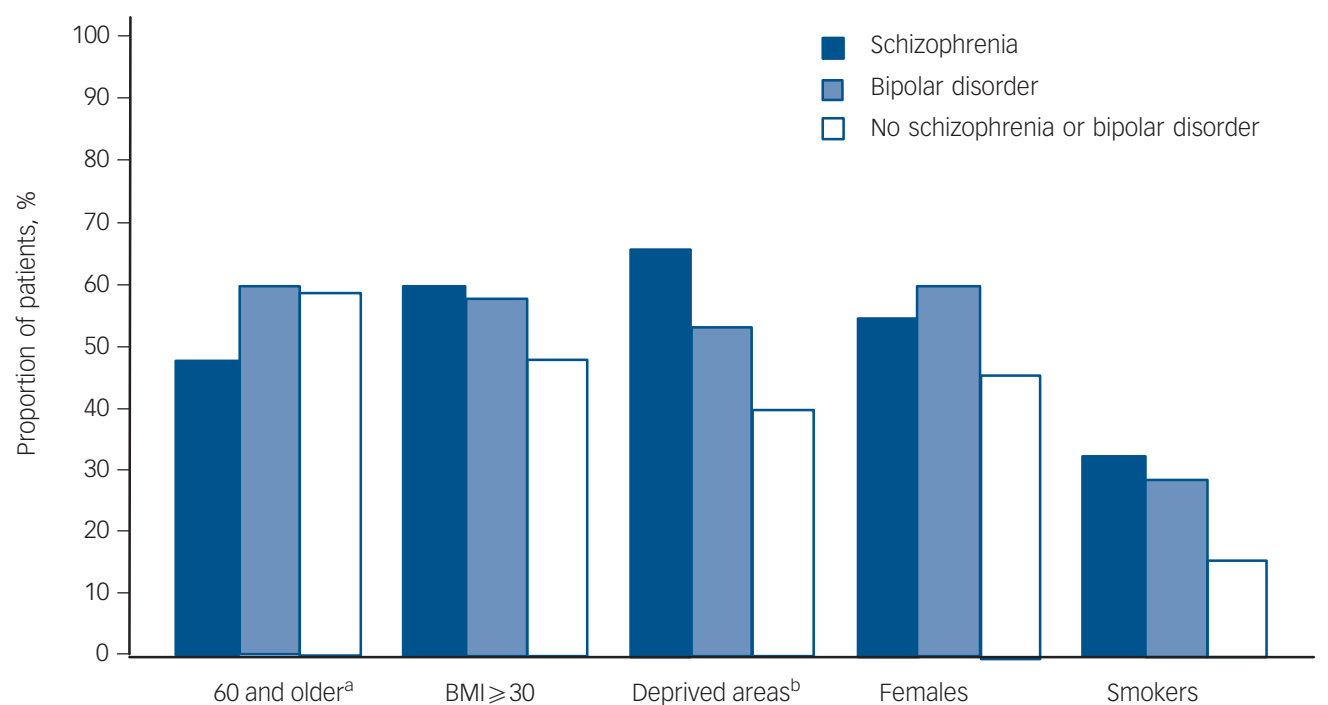

Fig. 1 Proportion of individuals with basic characteristics.

a. Age 60 or older at diagnosis of diabetes.

b. Deprived areas with Townsend score fifths of 4 and 5 .

The adjusted hazard ratios were 1.52 (95 CI 1.17-1.97) for schizophrenia and 1.47 (95\% CI 1.07-2.02) for bipolar disorder. After additional adjustment for overall use of antipsychotics, the effects of schizophrenia and bipolar disorders on mortality were diminished but remained statistically significant (adjusted odds ratios $1.38,95 \%$ CI $1.06-1.80$ and $1.41,95 \%$ CI $1.03-1.94$ respectively).

Figure 2 shows the estimated survival proportions of over a number of years based on a diagnosis of diabetes at 60 years of age. The estimates were based on the model where only gender 
Table 2 Crude survival rates $(95 \% \mathrm{Cl})$ after diagnosis of diabetes

\begin{tabular}{|lccc|} 
& \multicolumn{2}{c}{$(95 \% \mathrm{Cl})$} \\
\cline { 2 - 4 } & $\begin{array}{c}\text { With schizophrenia } \\
(n=257)\end{array}$ & $\begin{array}{c}\text { With bipolar disorder } \\
(n=159)\end{array}$ & $\begin{array}{c}\text { No schizophrenia or bipolar disorder } \\
(n=43589)\end{array}$ \\
\hline Crude survival rates at 5 years after diabetes diagnosis & $79(69-87)$ & $91(80-96)$ & $82(81-83)$ \\
\hline Crude survival rates at 10 years after diabetes diagnosis & $63(53-71)$ & $60(49-70)$ & $65(64-67)$ \\
\hline
\end{tabular}

Table 3 Hazard ratios $(95 \% \mathrm{Cl}$ ) for mortality during follow-up for people with schizophrenia and bipolar disorder between 1 January 2000 and 1 April 2005

Hazard ratios $(95 \% \mathrm{Cl})$

Unadjusted Adjusted for age $\mathrm{e}^{\mathrm{a}}$ and gender $\quad$ Adjusted for all factors ${ }^{\mathrm{b}}$

$\begin{array}{lcc}\text { Individuals without schizophrenia or bipolar disorder } & 1.00 & 1.00 \\ \text { Individuals with schizophrenia } & 1.21(0.93-1.57) & 1.84(1.42-2.40) \\ \text { Individuals with bipolar disorder } & 1.30(0.95-1.78) & 1.51(1.10-2.07) \\ \text { a. Age at diagnosis of diabetes. } & \\ \text { b. Adjusted for age at diagnosis of diabetes, gender, smoking status, deprivation, obesity, use of statins. } & 1.52(1.17-1.97)\end{array}$

and age were taken into account (Table 3). Survival rates were lowest for the people with schizophrenia, then for the group with bipolar disorder and were highest for those without these mental health problems.

\section{Discussion}

\section{Findings}

In line with previous research, ${ }^{14-16}$ these results show that people with diabetes who also had schizophrenia or bipolar disorder, were more likely to live in more deprived areas, to be smokers and to be female than those who did not have these disorders. Individuals with schizophrenia or bipolar disorder had a lower mean systolic blood pressure, which could be explained by the younger age and higher proportion of women. ${ }^{17}$ People with schizophrenia were also younger at diagnosis of diabetes and had a higher body mass index.

An analysis of Kaplan-Meier estimates of survival rates showed no significant differences between those with and without the mental disorders. However, people with schizophrenia were younger and those in both groups were more likely to be female so, after adjusting for age and gender, individuals with diabetes and schizophrenia or bipolar disorder had a significantly increased risk of death compared with those with diabetes alone. Adjusting for additional confounding variables (smoking status, deprivation, obesity, use of statins) slightly reduced the risk of death in people with schizophrenia and diabetes, but it still remained 50\% higher (the adjusted hazard ratio was 1.5) than for those with diabetes alone. The risk of death for people with bipolar disorder adjusted for age and gender was 50\% higher than for those without these mental health problems and changed very little after adjusting for the other confounders.

We have shown that people with diabetes and schizophrenia or bipolar disorder have higher mortality rates than individuals with diabetes alone. There are several possible explanations for this difference. In people with schizophrenia or bipolar disorder, diabetes may progress more rapidly, it may be more poorly controlled, or these individuals may have more comorbid physical illnesses and therefore be more likely to die of other causes.

In the UK, it seems unlikely that people with schizophrenia or bipolar disorder now have more poorly controlled diabetes. A
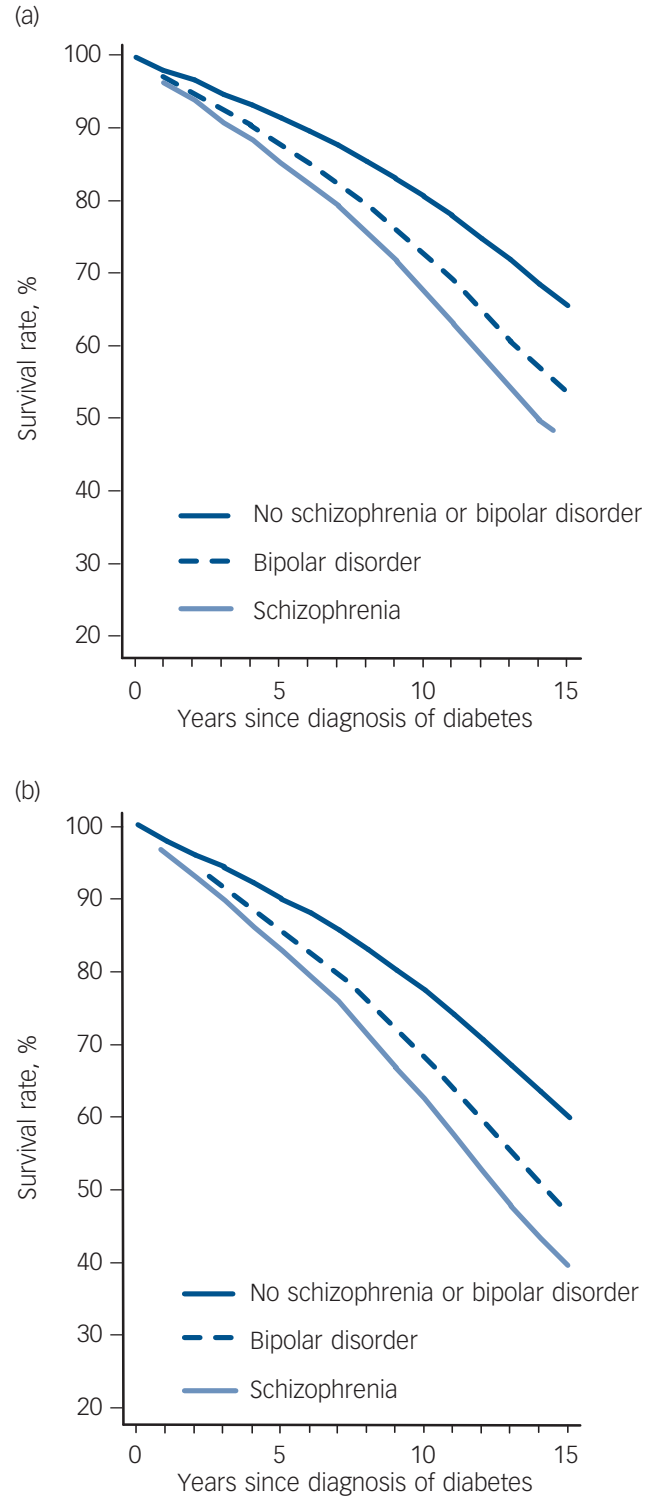

Fig. 2 Estimated survival rates in (a) women and (b) men with diabetes at 60 years of age. 
recent study was conducted as part of the same Disability Rights Commission project ${ }^{16}$ as this study and used a similar sample derived from the same database. It found that, since the introduction of the new General Medical Services contract for general practitioners on 1 April 2004, diabetes care in the UK has been as good for people with schizophrenia or bipolar disorder as for those without and, in particular, that they had as good or better glucose control as measured by $\mathrm{HbA}_{1 \mathrm{c}}$. The new contract was, however, introduced towards the end of the period from which data for this study was collected so it is possible that prior to its introduction diabetes care was worse for those with schizophrenia or bipolar disorder, partly or wholly accounting for the difference in mortality seen in this study.

It seems more likely that the increased mortality rate is related to greater rates of comorbid physical illnesses in individuals with schizophrenia and bipolar disorder. This has been found in other studies $^{1,18}$ and, in relation to chronic obstructive pulmonary disease, stroke, coronary heart disease and certain cancers, in some studies associated with the Disability Rights Commission project. $^{10,19}$

\section{Limitations}

This study only included people who were registered with a GP, so it cannot comment on people who do not access primary care and who may have different rates of diabetes, schizophrenia or bipolar disorder. These include some prisoners and people with schizophrenia or bipolar disorder known to a community mental health team but not to primary care services. It also cannot comment on people who have diabetes, schizophrenia or bipolar disorder, but who have not been diagnosed as such. There are, for example, an estimated 600000 people in the UK with undiagnosed diabetes. ${ }^{13}$

A greater concern is that because people with schizophrenia and bipolar disorder have a shorter life expectancy, on average more of them might have died before being included in the study than might those with diabetes alone. It is, therefore, possible that the survivors are not typical of the whole population with schizophrenia or bipolar disorder.

The survival analyses were based on all-cause mortality rather than deaths attributed to diabetes. This avoids inaccuracy as a result of the known unreliability of recorded causes of death, but prevents differentiation between a diabetes-related cause of increased mortality and increased mortality due to comorbidity. A high proportion of the people with mental disorders in this study were prescribed antipsychotic drugs and it is possible that these might have increased or decreased their survival rates depending on the type of drug and its duration of use, as shown in a study of mortality in people with schizophrenia. ${ }^{20}$ However, we did not carry out a detailed analysis according to type of antipsychotic drug prescribed, dose or duration of use as this was not within the scope of this study, but, adjusting for the overall use of antipsychotics in the survival analysis did not explain the increased mortality in the groups with bipolar disorder or schizophrenia.

These findings demonstrate the importance of good-quality diabetes care for people with schizophrenia or bipolar disorder. Individuals with schizophrenia or bipolar disorder have both an increased prevalence of diabetes and lower survival rates after diabetes is diagnosed. Diagnosing and treating people with schizophrenia or bipolar disorder proactively and ensuring that they take advantage of the healthcare available to them is necessary to reduce the inequality in outcomes between those with and without these mental illnesses.
Yana Vinogradova, MSC, Carol Coupland, PhD, Julia Hippisley-Cox, PhD, MD, Division of Primary Care, University of Nottingham, University Park, Nottingham; Seán Whyte, PhD, Springfield Hospital, London; Catherine Penny, PhD, Institute of Psychiatry, London, UK

Correspondence: Yana Vinogradova, Department of Primary Care, Tower Building 13th Floor, University Park, University of Nottingham, Nottingham NG7 2RD, UK. Email: yana.vinogradova@nottingham.ac.uk

First received 23 Oct 2009, final revision 23 Apr 2010, accepted 14 Jun 2010

\section{Funding}

This study was funded by the UK Disability Rights Commission and by core funding from the University of Nottingham.

\section{Acknowledgements}

We thank the practices contributing to the QRESEARCH database and also acknowledge the technical expertise of David Stables (EMIS computing) in establishing the QRESEARCH database.

\section{References}

1 Harris EC, Barraclough B. Excess mortality of mental disorder. Br J Psychiatry 1998; 173: 11-53.

2 Brown S, Kim M, Mitchell C, Inskip H. Twenty-five year mortality of a community cohort with schizophrenia. Br J Psychiatry 2010; 196: 116-21.

3 Dembling BP, Chen DT, Vachon L. Life expectancy and causes of death in a population treated for serious mental illness. Psychiatr Serv 1999; 50: 1036-42.

4 Tsuang MT, Woolson RF, Fleming JA. Premature deaths in schizophrenia and affective disorders. Arch Gen Psychiatry 1980; 37: 979-83.

5 Expert Group. 'Schizophrenia and diabetes 2003'. Expert Consensus Meeting, Dublin 3-4 October 2003: consensus summary. Br J Psychiatry 2004; 184 (suppl 47): s112-4.

6 Morriss R, Mohammed FA. Metabolism, lifestyle and bipolar affective disorder. J Psychopharmacol 2005; 19 (suppl 6): 94-101.

7 Jackson C, Covell N, Drake R, Essock S. Relationship between diabetes and mortality among persons with co-occurring psychotic and substance use disorders. Psychiatr Serv 2007; 58: 270-2.

8 Hammersley V, Hippisley-Cox J, Wilson A, Pringle M. A comparison of research General Practices and their patients with other practices - cross sectional survey in Trent. Br J Gen Pract 2002; 52: 463-8.

9 Hippisley-Cox J, Hammersley V, Pringle M, Coupland C, Crown N, Wright L. How useful are General Practice databases for research? Analysis of their accuracy and completeness in one research network. Health Informatics $J$ 2004; 10: 91-109.

10 Hippisley-Cox J, Parker C, Coupland C, Vinogradova Y. Inequalities in the primary care of patients with coronary heart disease and serious mental health problems: a cross-sectional study. Heart 2007; 93: 1256-62.

11 Office for National Statistics. Geographic Variations in Health: 326-37. TSO (The Stationery Office), 2001.

12 Jenkins $R$, Bebbington $P$, Brugha TS, Farrell $M$, Lewis $G$, Meltzer $H$. British psychiatric morbidity survey. Br J Psychiatry 1998; 173: 4-7.

13 The Information Centre for Health and Social Care. Key Findings about the Quality of Care for People with Diabetes in England, incorporating Registrations from Wales. National Diabetes Audit. The Information Centre, National Clinical Auidt Support Programme, 2006.

14 Dickerson FB, Brown CH, Kreyenbuhl JA, Fang L, Goldberg RW, Wohlheiter K, et al. Obesity among individuals with serious mental illness. Acta Psychiatr Scand 2006; 113: 306-13.

15 Kelly C, Mccreadie RG. Smoking habits, current symptoms, and premorbid characteristics of schizophrenic patients in Nithsdale, Scotland. Am J Psychiatry 1999; 156: 1751-7. 
16 Whyte S, Penny C, Phelan M, Hippisley-Cox J, Majeed A. Quality of diabetes care in patients with schizophrenia and bipolar disorder: cross-sectional study. Diabet Med 2007; 24: 1442-8.

17 The NHS Information Centre for Health and Social Care. Health Survey for England - 2008 Trend Tables. Joint Health Surveys Unit, 2008.

18 Phelan M, Stradins L, Morrison S. Physical health of people with severe mental illness. BMJ 2001; 322: 443-4.
19 Hippisley-Cox J, Vinogradova Y, Coupland C, Parker C. Risk of malignancy in patients with schizophrenia or bipolar disorder: nested case-control study. Arch Gen Psychiatry 2007; 64: 1368-76.

20 Tiihonen J, Lönnqvist J, Wahlbeck K, Klaukka T, Niskanen L, Tanskanen $\mathrm{A}$, et al. 11-year follow-up of mortality in patients with schizophrenia: a population-based cohort study (FIN11 study). Lancet 2009; 374: 620-7.

\section{poems by} doctors

\section{What has to be becomes}

\section{Aarohee B. Desai}

An Incongruous,

Painful yet Pleasurable

Vague but Sensual

Silhouette of a Thought -

A Passion

Takes a Concrete Shape

A Concrete Form.

A Shape

Sensitive, Sanguine, Seductive

Yet Suave, Sensible, Serene

And Secret.

A Shape rendered Concealed -

Unrevealed -

By Norms, Laws \& Rules.

All Forms Alive,

All Sensually Perceivable Forms

Are a Well-Revealed Concrete Shape

Of a Supreme \& Original

Imagination \& Thought.

Nature, Creatures \& Man

A Concrete Prodigious Shape

of a Thought

The Exact Expression of Which

Remains Unscrupulous, Unhindered -

By Norms, Laws \& Rules.

Beauty in the Ugly

And Vulgarity in Beauty

Haziness in Clarity

Purity in Dirt

Brightness in the Dark

And Darkness in the Bright

Are Inseparable Facets

Of an Unscrupulous Prodigious

Creation of a Thought.

Man's Imagination - A Concept.

Man's Concept \& Deeds -

An Imagination's Concrete Shape.

No Imagination or Concept

Of a Bestowal can Embarrass, Shame or Harm;

Or justified in Concealment

By Rules, Norms \& Laws.

A Pregnant Thought

When Blooms in a Form \& Shape Concrete,

Even in Concealment -

Remains a Shape Concrete.

No Hardened Rules, Norms \& Laws

Work in Undoing the Done.

Not in the Long Run.

What Has to $\mathrm{Be}$, Has to $\mathrm{Be}-$

And Becomes!

Aarohee B. Desai is ST4 in General Adult Psychiatry, Manchester Royal Infirmary. 